\title{
Yield and Efficiency of FIT Tests Alone vs. In Combination with Risk Factors for Colorectal Cancer Screening
}

\author{
Vasu Sheel ${ }^{1,2}$, Leslie Azzis ${ }^{3,4}$, Rachel Hinrichs ${ }^{5}$, Thomas F. Imperiale Th,3,6 $^{1,3}$ \\ ${ }^{1}$ Indiana University School of Medicine; ${ }^{2}$ Indiana Clinical and Translational Sciences Institute; \\ ${ }^{3}$ The Regenstrief Institute; ${ }^{4}$ Ecole des Hautes Etudes de Santé Publique (EHESP); ${ }^{5}$ IUPUI \\ University Library; ${ }^{6}$ Roudebush VA Medical Center
}

Background: Although colonoscopy (CY) may be considered the best screening test for colorectal cancer (CRC), annual fecal immunochemical test (FIT), which quantifies fecal hemoglobin is a viable alternative. Countries and healthcare systems using FIT-based screening may need to prioritize which FIT positive persons requires CY sooner (e.g. within the same fiscal year). We conducted a systematic review of published literature to understand how the yield/positive predictive value (PPV) of FIT could be improved.

Study Design: We performed a search of electronic databases for articles published between 2015 and June 2020. Titles, abstracts, and full texts were independently screened. Included studies fulfilled predetermined criteria and had descriptive and quantitative data extracted. We identified studies comparing the yield of FIT for advanced colorectal neoplasia ([AN], CRC plus advanced adenomas) among FIT positive persons to the yield of AN when FIT is combined with risk factors (age, sex, BMI, etc.). Data were extracted to find yield and efficiency of FIT alone vs FIT plus risk factors among FIT positive persons.

Results: From 623 titles reviewed, 4 studies met inclusion criteria. The objective of the studies was to increase the yield of AN or CRC in FIT positive patients. The number needed to scope (NNS) among FIT positives to detect AN significantly decreased for each study when looking at high risk groups as yield/PPV increased. The yield with FIT alone ranged from $24 \%$ to $46 \%$ and the NNS from 2.2 to 4.1 . With risk factors, yield and NNS among those at high risk were $33.2 \%$ to $75.6 \%$ and from 1.3 to 3.0 , respectively.

Conclusion and Potential Impact: This systematic review quantifies how risk factors improve the yield for AN in FIT positive persons, which is information required for countries and health care settings with limited resources that need to direct CY resources to FIT positive patients at high risk for $\mathrm{AN}$. 